\title{
An Inverse Global Environmental Kuznets Curve ${ }^{\text {: }}$
}

Raghbendra Jha

Australian National University
K.V. Bhanu MURTHY

University of Delhi

\begin{abstract}
All correspondence to:
Prof. Raghbendra Jha, Australia South Asia Research Centre

Division of Economics

Research School of Pacific and Asian Studies,

The Australian National University

CANBERRA ACT 0200

Australia

Telephone: $\quad+61261252683$

Facsimile: +61261250443

Email:<r.jha@anu.edu.au>

JEL Classification Number: Q0, Q2, Q3

Keywords: Global Environmental Kuznets Curve, Principal Components, Environmental Degradation Index
\end{abstract}

\footnotetext{
${ }^{*}$ We are grateful to the MacArthur Foundation for financial support and John Whalley and D.V. Rukmini for helpful discussions.
} 


\begin{abstract}
This paper articulates the necessity of emphasizing the absolute level of environmental degradation in different countries as a guide to understanding the links between such degradation and economic development. We argue the case for developing a composite environmental degradation index (EDI) and relating it to a better measure (in comparison to per capita income) of economic development, viz. the HDI with a view to developing a Global EKC (GEKC) across 174 countries. We put forward a methodology that would achieve this end. This has necessitated the use of principal components analysis to extract information and for forming an index of global environmental degradation. This index has then been related to the HDI.

Some other conclusions of the paper are as follows. First, there seems to be an inverse link between HDI ranks and EDI. Second, the US alone has a highly disproportionate (adverse) influence on all the six environmental degradation indicators. Therefore, in the design of a regulatory mechanism for global environmental management this factor would have to be recognized explicitly.

In the extant literature there is no consensus about the empirical basis for global environmental degradation (GED). All along the two contending views only try to verify whether or not an inverted $U$ shaped EKC exists. Even if we agree that an inverted $U$ shaped EKC does not exist it does not follow that an inverted $N$ shaped global EKC exists. Our study argues precisely that an inverted $N$ shaped global EKC does indeed exist and provides empirical support for this position. In the process we discover extreme inequalities in the contribution of low, medium and high country groups to GED with the low country group effectively ameliorating GED and the high group countries exacerbating it.

There are several important implications of the results of this paper and a few of these may be mentioned here. First, it provides a framework for assessing the current state of environmental degradation and its distribution world-wide. Thus, it would provide important inputs to a global agency like a WEO that would be interested in monitoring environmental degradation and its geographical distribution. Second, by relating such degradation to HDI, this relationship would highlight the contributions of countries with different levels of human development and assist in assigning liability. The contention that GED is essentially 'caused' by a certain type of development characteristic of high (income) development countries is borne out by the results of this study.

These conclusions clarify that the first and foremost concern of global environmental management, and the WEO, should be to reckon with the inequality in global environment degradation and its intrinsic relationship with inequalities across countries in levels of economic development.
\end{abstract}




\section{Introduction}

The Environmental Kuznets Curve (henceforth EKC), is purported to be an inverted $U$ shaped curve designed to explain the interdependence between levels of economic development and environmental degradation ${ }^{1}$. This notion is sometimes described for any given country and the EKC defined as a relation between per capita income and select environmental indicators ${ }^{2}$. Thus, drawing from the experience of existing economically developed countries, it is argued that as a country's per capita income grows, income elasticity of demand for the environment will become positive and may rise to a value larger than one.

However, there has been little effort to relate per capita income (or some other broad measure of economic development) to a composite index of environmental degradation in a cross section of countries. Surely, such an effort is necessary to understand whether, at any given point in time, an EKC type relation can be found to be empirically valid. Jha and Whalley (2000) have argued that the notion of the EKC (typified as a relation between per capita incomes and select pollutants as in the extant literature) for any given country is tenuous, at best. Even if this relation has characterized the experience of any given country, a jump to conclude that it is universally valid without checking the cross section properties, is untenable.

In this paper we examine the underpinnings of this cross sectional (cross-country), global EKC (henceforth GEKC). While doing so, we use a more complete measure of

\footnotetext{
${ }^{1}$ It is so called because Kuznets (1955) had found a similar inverted - $U$ shaped relationship between income growth and income inequality.

${ }^{2}$ Some studies have done cross-country estimation. See Grossman and Krueger (1995), Shafik and Bandopadhya (1992) and Selden and Song (1994).
} 
economic development than per capita income - the Human Development Index ${ }^{\text {G }}$ (HDI) ranks of countries and attempt to relate these to their records of environmental degradation as captured in a composite Environmental Degradation Index (EDI). If the EKC has an inverted $U$ shape, as is often purported, then this kind of a relationship should be confirmed between these two indicators in a cross section of countries as well. This paper proposes to test this proposition.

The plan of the rest of this paper is as follows. Section II discusses the relationship between sustainability and $\mathrm{EKC}$, from the perspective of developing countries. Section III contains a conceptualization of 'global environmental degradation'. In Section IV the details of methodology for our empirical testing and data are given. Section V analyses the results. Section VI concludes.

\section{The Growth-Environment Debate}

There are two broad standpoints regarding the relationship between economic growth and environmental degradation. Some studies emphasize that the higher the income level greater would be the environmental degradation (Daly (1977), Georgescu-Roegen (1971)), Hall et. al. (1986)). However, Beckerman (1992,1993), GATT (1992), Bartlette(1994) and Panayotou (1993) believe that, after a point, income growth lowers environmental degradation. They point out that the relationship between environmental degradation and the income level is non-linear - positive upto a point and becoming negative after a threshold level of income has been achieved. We shall refer to this controversy as the "growth-environment debate"

\footnotetext{
${ }^{3}$ As is well known, the HDI rank is an ordinal index.

${ }^{4}$ This discussion is based on Cole (2000) but is extended, through Jha and Bhanu Murthy (1999), Jha and Whalley (2000) and Stagl (1999), so as to highlight the developing country perspective, because this paper is concerned about developing countries.
} 
One question that arises in this context is: how does mainstream economic theory and policy treat the issue of growth and environment. Economic growth theory almost ignores the environment. Where it does allude to the environmental effects of growth it prescribes that these could be dealt with separately or that they would take care of themselves (Arrow et. al. (1995)). Trade theory's approach is an anthropocentric view Trade policy is also concerned with environmental issues ${ }^{\natural}$ but from the narrow perspective of 'trans-boundary pollution', 'permits', and so on ${ }^{\text {! }}$.

The extant studies have, in the main, attempted to measure and analyze Individual (country) Environmental Kuznets Curves (IEKC). Grossman and Krueger (1995) carry out a cross-country study using a number of environmental variables. However, the data sets are non-uniform in that data on some variables referred to individual cities and, in the case of others, to countries. The coverage of data over the years is also not uniform. In Shafik and Bandopadhya (1992) a large number of variables were used to test for an EKC type relation in 149 countries but they studied only the effects of individual variables. In particular, there was no attempt to compute a composite index of these variables nor was there any attempt to model a global EKC. Selden and Song (1994) studied global pollution within a framework that distinguished between countries with high, low and medium incomes. They however restricted themselves to air pollution and did not make an attempt to articulate a composite index of environmental degradation. Janicke et. al. (1989) used an aggregate indicator for volume of throughput based on four indicators

\footnotetext{
${ }^{5}$ Trade is concerned with profit, trade and economic growth of human beings only and not the entire earth system (the biosphere), including all the species.

${ }^{6}$ See Antweiler (1998) and Esty (1994) on free trade and the environment. Also see, Suri and Chapman (1998) on trade pattern and EKC.
} 
relating to energy, steel and cement and rail and road freight. But it was limited to 31 COMECON and OECD countries and covered the period 1970-85. They confirmed an EKC relationship. Later this was challenged by the 'relinking' hypothesis. Birdsall and Wheeler (1991) studied air pollution in the manufacturing sector of Latin America using developed and developing country data. They concluded that pollution intensity measured as the ratio of air pollution to GDP declines with income in open economies and rises in relatively closed economies.

This enquiry would be incomplete without a discussion on the relation between EKC and sustainable development. Pearce (1993) states that all versions of `sustainable development' are based on the principle of equity. In the seventies, the growthenvironment debate was dominated by approaches based on the Entropy Law 8 (Daly (1973), Georgescu-Roegen (1971)). The physical entropy models rest on a global view of physical stocks of matter and flows of energy. This approach was opposed to the 'growthmania' and advocated the establishment of a 'steady state', that not only implied a constant capital stock but also a ‘constant stock of people' (Daly(1973) p.15). Daly questions not only the feasibility of growth but also its desirability. Such a view is clearly anti-developmental. The problem is that the truth of the entropy models cannot be ignored, since they are based on physical phenomena. However these models ignore the distributive issues of growth and environment, which is the crux of the matter. Developing countries are already at a low level of economic development. Now they are

\footnotetext{
${ }^{7}$ Some studies attempt to relate the EKC to trade issues (Hettige et. al. (1992) )

${ }^{8}$ It is also known as the Second Law of Thermodynamics. Rees (1990) clarifies that "The Second Law states that in any closed isolated system, available energy and matter are continuously and irrevocably degraded to the unavailable state". (Also see (Daly and Townsend (1993), Georgescu-Roegen (1971) and Ekins (1993)).
} 
being asked to contain their rate of development. The implication of such models is that since present day developing countries possess natural stocks they must pay the price for the depleted global 'free energy' (low entropy), while the developed world has already amply benefited and has been largely responsible for the depletion. In so far as the pattern of development of advanced countries continue to burden the global environment and in so far global environmental management needs 'global solutions', this inequality is likely to be perpetuated and the prospect for the developing world shall remain dismal.

A crucial question that arises is when, and in what form, world bodies responsible for development and global management, have taken to the issue of growth and environment. As opposed to the seventies, in the eighties, the concern turned towards “sustainable development”(World Commission on Environment and Development (1987) (Brundtland Report) ${ }^{2}$. Ekins (1993) points out that it is only thereafter that chambers of business and world bodies started taking interest in the issues concerning sustainability for the first time $\mathrm{L}^{10}$. However, both independently believed that economic growth provides the conditions in which protection of the environment can best be achieved. Unfortunately, even this stand does not augur well for developing countries because it must be seen in the context of the EKC, which tells them that, as long as they are below the threshold of development, growth would only increase their environmental degradation. However, this approach implies that they must sacrifice growth for the sake of a healthy global environment. This means that global intertemporal efficiency is

\footnotetext{
${ }^{9}$ Also see Ruttan (1994).

10 'Business Charter for Sustainable Development' (International Chamber of Commerce, 1990) and ' Business Council for Sustainable Development' (UN Council for Environment and Development, 1992). Thereafter, the World Bank (1992) and the WTO.
} 
sought to be achieved through global atemporal (spatial) inequity. We believe that "the applicability of the notion of sustainability has ultimately got to be universal and refer to the indefinite future" and must be related to consumption 11 (Jha and Bhanu Murthy

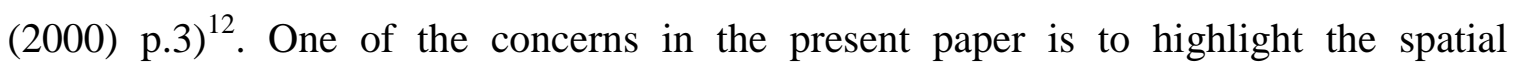
dimension of sustainability, in relation to economic development. Both the sides of the debate fail to highlight this dimension $\frac{13}{\text {. }}$

Even later developments, which have been broadly classified as 'weak' and 'strong' sustainability approaches, do not focus on the spatial dimensions of environment and the special problems of developing countries. The argument of the 'weak sustainability approach' is that while growth entails environmental degradation either the levels of degradation would fall after a threshold (in the EKC) or degradation could be de-coupled or de-linked from growth $\frac{14}{14}$ Subsequent studies attempt to detail the manner in which this de-linking is possible $e^{15}$. Bernstam (1991) attempts to link the fate of the South to that of the North. He argues that economic growth in the South depends on growth in the North. Hence accelerated growth in the North would result in the South surmounting the EKC hump. This argument is 'anti-developmental' because it indirectly justifies the existing gap between the North and South and provides a rationale for further accelerating the growth of the North.

\footnotetext{
${ }^{11}$ Rothman (1998) also makes out a case for a consumption-based approach to the EKC.

${ }^{12}$ A number of definitions of sustainability are discussed here, ibid. p. 4- 8.

${ }^{13}$ Some studies have, however, attempted to highlight the relationship between poverty and the environment (Broad (1994)).

${ }^{14}$ World Bank (1992) first used this term.
} 
There is in general a tendency in the literature to take the inverted $U$ - shape of EKC for granted and then proceed to give explanations for it. Apart from the above problems in accepting an apparently optimistic position taken by the 'weak sustainability theorists', there are some pertinent arguments relating to EKC that have been raised by Stern et. al. (1996), Stern (1998) and Ekins (1997), amongst others. The arguments as well as the counter-arguments are listed below.

1. There may be a negative feedback from environmental degradation to income growth, especially in the case of LDCs. This may be the cause of the inverted U- shape. However, Holtz-Eakin and Selden (1992) find no evidence of such simultaneity.

2. North exports its pollutants to the South, therefore, we may have the purported inverted $U$ shaped EKC. Once again, this argument rests crucially on the evidence to support the inverted $U$-shape.

3. Ekins(1997) argues that OECD countries have passed the hump of emission. A casual observation of data in this study suggests the opposite.

4. Some econometric issues have also been raised. For instance Stern et. al. (1996) feel that corrections for heteroscedasticity have not been made. Our study has attempted to address the problem.

Amongst others Harbaugh et. al. (2000) find no evidence of EKC relationship. And, Moomaw and Unruh (1997) and Bergh (1998), suggest that what might appear as delinking may not persist in the long-run. Finally, there is the re-linking hypothesis ${ }^{16}$ that purports an $N$ - shaped curve, wherein environmental degradation is once again

\footnotetext{
${ }^{15}$ Ekins (1997), de Bruyn (1997), de Bruyn and Opschoor (1997) - attribute de-linking to at least, de-pollution and dematerialization.

${ }^{16}$ Pezzy (1989) and de Bruyn et. al. (1998).
} 
positively linked to income levels. Here the question is whether it is a straight $N$ or an inverted $N$.

\section{The Phenomenon}

What would happen if the logic of the IEKC were extended to GEKC? The GEKC would purport that at low income levels environmental degradation rises with the income level and at high levels of income there is a decline in the environmental degradation (like an inverted $U$ curve). This argument, however, makes it appear as though the LDCs are responsible for environmental degradation because the EDI rises with income in their case. By the same token it makes it appear as though the developed (rich) countries reduce environmental degradation. What is critical here, however, is the level of per capita income at which the hump in the GEKC occurs. Assume that the GEKC exists and that developed countries are on the right side of the hump. Even then, given the large gap between the HDI of developed and developing countries, the absolute level of EDI is likely to be much higher in the case of developed countries as compared to developing countries. In any event, this is a proposition to be tested. The extant literature on EKC has emphasized the direction of change of EDI with respect to per capita income, not its absolute level. In fact two studies confirm such a rise in the absolute level. As opposed to Birdsall and Wheeler (1991) Lopez (1994) found that even in the most open economies, while the pollution intensity falls the absolute level of pollution increases with income. Second, Hetttige et. al. (1997) actually found that absolute levels of water pollution rise through the middle income countries and remain stable thereafter.

A number of questions emerge when considering the EKC. Firstly, is it a local phenomenon restricted to individual countries or is it a global phenomenon? Secondly, is 
the EKC uniformly declining or an inverted $U$-shaped curve as is suggested in literature? The implications in each case are vastly different. Thirdly, is income an appropriate basis for tracing the EKC? Hence there is a need for a fresh examination of the empirical form and analytical content of the GEKC.

If EKC is a purely local phenomenon, then the question arises whether this construct is independent in each country. Is one country's EKC related to other countries' EKC? If one admits the possibility of a Global EKC then one has to probe its underpinnings. A maintained hypothesis of the present paper is that global environmental problems are rooted in local phenomena. If this were true then the GEKC would arise within a collective cross-sectional (cross-country) framework. An obvious supplementary enquiry would be into the shape of the GEKC.

A major issue with regard to the EKC is that extant studies have taken for granted the conceptual phenomenon of 'global environmental degradation' (GED) and its empirical basis. This has resulted in two practices while modeling this phenomenon. The first is the use of the regression framework without adequate justification and second is the method of relating individual degradors with individual (and perhaps partial) indicators of economic development. At least a part of the problem arises from not delving into the data generating processes, i.e., the factors responsible for the phenomenon, their characteristics, the inter-relationships and most of all, the appropriate level(s) for the empirical study. As for the phenomenon per se, often GED has been treated as a geographic and natural phenomenon and not explicitly as an economic phenomenon, more particularly one that arises out of a 'type of economic development'. Our approach is to study the phenomenon at the level of country groups, as well as, 
globally, for all countries and all degradors. The preferred method is to relate a composite index of GED with HDI, a composite index of economic development (as opposed to certain countries and certain degradors). This enables us to understand the phenomenon of GED as an economic phenomenon (rather than a geographic and natural phenomenon), that is being 'caused' by certain 'latent' factors, related to economic development. Factor analysis allows such phenomena to be gauged on the basis of latent factors. In fact, it allows for alternative, plausible sets of latent factors for explaining the same phenomenon (of say, GED). While the same could be said of many economic phenomena, it is truer of GED that alternative latent factors may be responsible for it.

Coming back to the question of GED, and the need for an appropriate empirical framework, two things need to be said. First, that the ideal framework would be to conceptualize GED as a "composite" since it would be simplistic to assume otherwise. And second, that in so far as real world economic phenomena need to be gauged by inductive methods rather than deductive methods (which may, nevertheless, be appropriate for physical phenomena) some amount of exploratory empiricism is called for. Factor analysis allows for such exploratory and confirmatory analysis. Further, such exploratory empiricism would not only help in gathering the contours of the phenomenon of GED but would also reveal the weakness of the regression analysis in general, and in particular, the weakness of the usual procedure of isolating some pollutants and attempting to relate these to per capita income. The exploratory empirical exercises are listed in the relevant section below but the concept of "composite" needs to be clarified before attempting to conceptualize the phenomenon of GED. Here it may be said that a composite is a conglomerate of many factors that may be acting as vectors in different 
directions, with the resultant vector having a certain central tendency (the grand mean). A secular increase in this tendency would be indicative of the phenomenon of GED. The composite of GED is in this sense, 'caused' by another composite of economic development where each of the composites is appropriately weighted. It is, in this sense, inappropriate to isolate one indicator of each (pollution and level of development) and attempt to explain the phenomenon of GED by relating the two. This method would not only be biased but would also inconsistent. Biased because while GED and economic development are composites, each country group (high, low or medium) would have specific predominating characteristics with regard to GED incorporated in a specific indicator. Inconsistent because firstly, the choice of different factors would successively give varying results and secondly, because isolating one factor and embedding it in a regression framework would result in such inconsistency. This is especially true because three problems may be expected of the regression framework: a. Multi-collinearity; b. Simultaneity; and c. Loss of information.

An examination of the above problems reveals the weakness of regression analysis and the limitations of isolating individual pollutants for relating them to per capita income, which is the usual methodology employed. Some exploratory empirical exercises in this regard also facilitate the understanding of the phenomenon of GED. Such an analysis throws up the following leading questions. How does GED take place? In this respect, if the intention is to study the composite phenomenon, all factors 
responsible for GED must be included in the analysis. Why is GED a composite? What are the implications of these questions for methodology 17 .

GED occurs as a result of an accumulation of local phenomenon such as:

Pollution - of various types; b)Lack of bio-diversity; c) Waste- toxic and non-toxic; and d) Erosion of the natural resource base due to phenomenon like deforestation, depletion of fresh water resources, paper consumption, etc. The above set of indicators could be called 'state of the world'.

GED is a composite because such phenomena mutually influence each other. For instance, excessive paper consumption would result in deforestation, which would cause a fall in water resources and a growth in $\mathrm{CO} 2$ levels, which would then cause global warming, soil degradation and denudation, which would adversely affect bio-diversity and so on. Therefore, it is not possible to separate cause and effect. Hence, we do not treat these factors exclusively either as causes or effects. We would prefer to call them indicators of GED. In our understanding, the composite of GED is caused by a type of development captured by a composite index of HDI. At once the indicators suffer from simultaneity and multi-collinearity. However, most studies take up individual indicators (say $\mathrm{CO} 2$ levels) and attempt to explain their behaviour independently of the other indicators. Here again, there are two problems; firstly, $\mathrm{CO} 2$ levels are taken to be an effect of economic development, rather than 'a cause' of GED. Secondly, it is highly likely that the movements in the other indicators (auxiliary regressors) would be causing movements in $\mathrm{CO} 2$, while the same would be attributed to per capita income. The first implication for methodology is that a regression framework cannot be accepted without

\footnotetext{
17 "Trans-boundary pollution has been overemphasized in literature, as the cause of GED. So it must be pointed out that it is responsible only for the spread of pollution and would nevertheless remain only one of
} 
rigorously testing for multi-collinearity. The second implication is that the problem with multi-collinearity is not whether it exists but its degree. The most reliable method for testing for multi-collinearity is to carry out $k$ auxiliary regressions if there are $k$ such indicators, with each of the regressors being treated as regressands. This points out to the weaknesses of the regression framework of analysis in such a situation. For understanding these weaknesses, it is essential to study the formal algebra that forms the basis of such regression analysis.

The essential ingredients of a linear regression are a regressand $\mathbf{y}$ and a matrix of regressors $X=\left[\mathbf{x}_{1}, \mathbf{x}_{2} \ldots \mathbf{x}_{\mathbf{k}}\right]$. The regressand $\mathbf{y}$ is an $\mathrm{n}$ vector and the matrix of regressors $\mathbf{X}$ is an $\mathrm{n} x \mathrm{k}$ matrix with each column $\mathbf{x}_{\mathbf{i}}$ being an $\mathrm{n}$ vector. The regressand $\mathbf{y}$ and each of the k regressors can be thought of as points in the $\mathbf{n}$ dimensional Euclidean space, $\mathbf{E}^{\mathbf{n}}$. The k regressors span a k-dimensional subspace of $\mathbf{E}^{\mathbf{n}}$, denoted by $\boldsymbol{\delta}(\mathbf{X})$. If $\boldsymbol{\gamma}$ is a $\mathrm{k}$ vector then all points $\mathbf{Z}$ in $\mathbf{E}^{\mathbf{n}}$ constitute the subspace $\boldsymbol{\delta}(\mathbf{X})$ such that $\mathbf{Z}=\boldsymbol{\gamma} \mathbf{X}$. The dimension of $\boldsymbol{\delta}(\mathbf{X})$ is $\mathbf{r}(\mathbf{X})$, which is the rank of $\mathbf{X}$. The problem arises when the $k$ regressors are not linearly independent. They would then span a subspace of a dimension less than $k$, say $\mathrm{k}^{\prime}$, where $\mathrm{k}^{\prime}$ is the largest number of columns of $\mathbf{X}$ that are linearly independent. In such a situation, $\delta(\mathbf{X})$ will be identical to $\delta\left(\mathbf{X}^{\prime}\right)$, where $\mathbf{X}^{\prime}$ is an $n$ $\mathrm{x} \mathrm{k}^{\prime}$ matrix. In an extreme situation, all $\mathrm{k}$ regressors may be mutually dependent. Here $\mathbf{X}$ reduces to an $n$ vector. In a more realistic situation, it may be possible that pairs of $\mathbf{x}_{\mathbf{i}}$ 's are linearly independent, so we would have subspaces denoted by $\delta\left(\mathbf{x}_{1}, \mathbf{x}_{2}\right), \boldsymbol{\delta}\left(\mathbf{x}_{1}, \mathbf{x}_{3}\right)$ etc. But each of these subspaces is defined by the two vectors jointly. Hence, the regressand $\mathrm{y}$ is to be explained either by a single variable which makes the model highly restricted 
and deterministic or by sets of variables that are linearly independent, which would introduce an ambiguity because $\boldsymbol{\delta}(\mathbf{X})=\boldsymbol{\delta}\left(\mathbf{x}_{1}, \mathbf{x}_{2}\right)=\boldsymbol{\delta}\left(\mathbf{x}_{1}, \mathbf{x}_{3}\right)$..... Moreover, explaining $\mathbf{y}$ in terms of a linear transformation of regressors incorporated in one of the auxiliary regressions assumes exact multi-collinearity. If this were not be to the case, that is, if sampling variations are allowed for, there would be a 'loss of information' because of

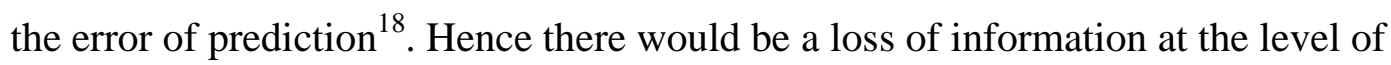
predicting regressand y also. Apart from multi-collinearity a limitation of the regression framework is the need for assuming normality. From the point of view of removing the three weaknesses of regression analysis, Principal Component Analysis (PCA) scores very well. Firstly, PCA is based on a linear transformation of the regressors such that they are orthogonal to each other by design. Hence, any information contained in the points in the event space is not lost. Secondly, the normality assumption is not essential, because, in a real world situation where there are wide differentials amongst countries, and between individual effects of indicators, such an assumption is dispensable. Thirdly, with such a dispersed set of outcomes, PCA is ideally suited because it maximizes the variance rather than minimizing the least square distance.

\section{Data and Methodology}

In this paper we attempt to estimate an GEKC for 174 countries represented in the latest Human Development Index (HDI) (UNDP (1999)). The data used in the HDI is cross section (cross-country) with most of the variables referring to the period 1990-1996. The HDI reports data on the following environmental variables $\frac{19}{1}$.

a. Internal renewable water resources per capita (cubic meters/ year) (1998);

\footnotetext{
${ }^{18}$ It can be shown that the error of prediction in general is orthogonal to the regression line

${ }^{19}$ The precise years for which the HDI reports the data are shown within parentheses against each category.
} 
b. Annual fresh water withdrawals per capita (hundred cubic meters) (most recent year within 1987-95 for which data is available);

c. Annual fresh water withdrawals as a percentage of water resources (most recent year within 1987-95 for which data is available);

d. Average annual rate of deforestation (\%) (reference period 1990-95);

e. Printing and writing paper consumed per 1000 persons; (1996)

f. Total CO2 emission (million metric tons) (1996);

g. Share of world total CO2 (\%) (1996);

h. Per capita CO2 emissions (metric ton) (1996);

i. SO2 emissions per capita (kilograms) (1995).

Data on $\mathrm{SO} 2$ was scanty so it was dropped. Internal renewable water resources per capita are very large in comparison to the other variables. Hence it as also dropped. For a similar reason the variable "total $\mathrm{CO} 2$ emissions" was also dropped. Thus we are left with six variables, that were named as:

1. PCFWW - Annual per capita fresh water withdrawals.

2. CENTFWW - Annual fresh water withdrawals as a percentage of water resources.

3. PAPCPM - Printing and writing paper consumed per capita.

4. $\mathrm{PCCO} 2$ - Per capita $\mathrm{CO} 2$ emission.

5. $\mathrm{CO} 2 \mathrm{SH}$ - Share of world total $\mathrm{CO} 2$.

6. DEFOR - Rate of deforestation.

While the above six variables were used for constructing a composite index, there could be additional indicators of GED such as biodiversity, waste and soil degradation. 
Paucity of comparable data prohibits us from using these variables. The selected variables were expressed as ratios or as per capita measures. This was done so as to minimize the scale problem. In certain cases, DEFOR was expressed in negative terms implying reforestation. Some of the data gaps (though they were very few) were filled with help of substitute means based on neighboring countries 20 .

The 174 countries covered by the HDI have been classified into three classes according to the following criteria:

a. Human Development Index $\geq 0.8$ - High Human Development;

b. Human Development Index 0.5 to 0.799 - Medium Human Development;

c. Human Development Index <0.5 -Low Human Development;

Accordingly, the following ranges represent different development classes:

a. Countries from HDI rank 1 - 45 - High Human Development;

b. Countries from HDI rank 46-139 - Medium Human Development; and

c. Countries from HDI rank 140 - 174 - Low Human Development.

\section{Exploratory empirical analysis}

One of the exploratory empirical exercises consisted of carrying out a two-factor analysis at two levels, one at the overall level and the other in respect of the three developmental classes, namely, low, medium and high HDI countries. This study was done with the help of analysis of variance (ANOVA- two factor (without and with

\footnotetext{
${ }^{20}$ SPSS package was used for estimation. It provides for substitute means being used for missing values. Neighboring data points were used for generating these substitute means. In any case, there were very dew missing data points.
} 
replication $\underline{21}$ ). The two factors are (a) 174 countries and (b) six environmental degradors. The two two-factor models that have been used are given in equations 1 and 2:

$$
X_{j k}=\mu+\alpha_{j}+\beta_{k}+\varepsilon_{j k}
$$

Here, $\mathrm{X}_{\mathrm{jk}}$ is a measure of environmental degradation due to the $\mathrm{k}_{\text {th }}$ degrador (column) and the $\mathrm{j}_{\text {th }}$ country (row). The random variable $\mathrm{X}_{\mathrm{jk}}$ is normally distributed across the grand mean $\mu$ with three additive effects;

i) $\quad \alpha_{j}$ - the country effect;

ii) $\quad \beta_{\mathrm{k}}-$ the degrador effect; and

iii) $\quad \varepsilon_{\mathrm{jk}}-$ the random effect.

The random effect is normally distributed while the total variance (V) is $\mathrm{V}=\mathrm{V}_{\mathrm{R}}+\mathrm{V}_{\mathrm{C}}+\mathrm{V}_{\mathrm{E}}$

That is, the total effect $V$ is the sum of the country effect (between country variance $\left(V_{R}\right)$ ), the degrador effect (between degrador variance $\left(V_{C}\right)$ ) and the overall random variance $\left(V_{E}\right)$. Their respective mean square errors were used to test whether either of these effects was statistically significant.

$$
X_{j k l}=\mu+\alpha_{j}+\beta_{k}+\gamma_{j k}+\varepsilon_{j k l}
$$

Here, $\mathrm{X}_{\mathrm{jk}}$ is a measure of environmental degradation due to the $\mathrm{k}$ th degrador (column), the $\mathrm{j}_{\text {th }}$ country-group (row - block) and. The random variable $\mathrm{X}_{\mathrm{jk}}$ is normally distributed across the grand mean $\mu$ with four additive effects;

i) $\quad \alpha_{j}-$ the country-group effect;

\footnotetext{
${ }^{21}$ The interactive model had to be restricted to 35 observations per country group because that was the maximum number of countries in the smallest group, i.e., and the low HDI country group.
} 
ii) $\quad \beta_{\mathrm{k}}-$ the degrador effect;

iii) $\quad \gamma_{\mathrm{jk}}-$ the group-degrador interaction effect; and

iv) $\quad \varepsilon_{\mathrm{jkl}}-$ the random effect.

The random effect is normally distributed while the total variance (V) is

$\mathrm{V}=\mathrm{V}_{\mathrm{R}}+\mathrm{V}_{\mathrm{C}}+\mathrm{V}_{\mathrm{I}}+\mathrm{V}_{\mathrm{E}}$

Thus, the total effect $V$ is the sum of the country-group effect (between country-group variance $\left(V_{R}\right)$ ), the degrador effect (between degrador variance $\left(V_{C}\right)$ ), interaction effect (variance $\mathrm{V}_{\mathrm{I}}$ ) and the overall random variance $\mathrm{V}_{\mathrm{E}}$. Their respective mean square errors were used to test whether either of these effects was statistically significant.

The above analysis reveals that both at the overall level and the country classes level, the "country effect" was not significant. On the other hand, the degrador effect was significant at both levels. However, in the case of low HDI countries, both the effects were not significant. (See Tables 1-3). This implies that it is meaningful to attempt the estimation of a global EKC across the entire set of 174 countries. An analysis of variance carried out for all countries together revealed that only the degrador effect is significant. (See Table 4). The model designed to capture interaction effects between country groups and degradors was not significant. However, the country group effect and the degrador effect were significant in this model. (See Table 5). As for the degrador effect, its being significant does not hamper the attempt to develop a composite index precisely because the methodology for constructing the index takes care of these differentials by using appropriate weights.

Tables 1 to 5 here. 
In the face of the country effect being insignificant, the question about the absolute levels of degradors arises. More precisely, if there is no country effect, are there differences between the mean levels of degradors across country classes? To examine this question, the mean and co-efficient of variation of the degradors respectively were estimated for each of the three country classes. The basic descriptive statistics (Tables 6 and 7) reveal that even though the country effect is not significant, the mean levels of degradors are highly co-related with the level of HDI. The high HDI country class has the highest mean values in all but one variable, that is, deforestation, which happens to be negative in their case $\mathrm{e}^{2}$. The medium HDI countries display middle range values, while the low HDI countries have extremely low values. Significantly again, the co-efficient of variation almost follows the reverse pattern, i.e., the behaviour of high HDI countries that display high levels of degradation is consistent because their co-efficient of variation is low in most cases. This gives a preliminary pointer to a negative relationship between environmental well being and level of economic development.

\section{Tables 6 and 7 here.}

We tested for multicollinearity among the regressors using auxiliary regressions. (See Table 8). An F-test was applied for testing for the overall significance of the $\mathrm{R}^{2}$ of the auxiliary regressions. All the $F$ tests reveal that multicollinearity was significant in all these auxiliary regressions. This validates the conceptualization of the environmental variables as indicators rather than independent regressors in a regression framework and underscores the need for developing a composite index.

\section{Table 8 here.}

\footnotetext{
${ }^{22}$ This is easily explained given that deforestation in these countries occurred at a much earlier time.
} 
One aim of our empirical work was to evolve a composite index of environmental degradation (Environment Degradation Index - EDI) and to relate it to HDI ranks so as to develop an GEKC. Here, we need to choose the essential variables and arrive at relative weights for the purpose of consolidating these variables into a single index. We chose Principal Components Analysis (PCA) which is popular in the literature since it has a number of desirable properties, as have been discussed above.

\section{Principal Component Analysis (PCA)}

This is a statistical technique that linearly transforms an original set of variables into a smaller set of uncorrelated variables that represents most of the information in the original variables

The methodology of PCA is as follows: Consider $p$ random variables $-\mathrm{x}_{1}, \mathrm{x}_{2}, \ldots$, $\mathrm{x}_{\mathrm{p}}$

$$
y_{1}=a_{11} x_{1}+a_{12} x_{2}+\ldots+a_{1 p} x_{p}=\sum_{i=1}^{p} a_{1 i} x_{i}
$$

The first principal component $\mathrm{y}_{1}$, is defined as

such that the variance of $y_{1}$ is maximized subject to the constraint that the sum of squared weights is equal to 1 , i.e.,

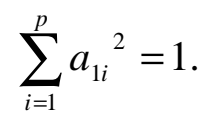

If the variance of $\mathrm{y}_{1}$ is maximized then the sum of the squared correlations with the original variables is also maximized. This is written as: 


$$
\sum_{i=1}^{p} r_{y, x i}^{2}
$$

PCA finds the optimal weight vector $\left(a_{11}, a_{12, \ldots} a_{1 p}\right)$ and the associated variance of $y_{1}$ which is denoted as $\lambda_{1}$.

The second principal component $\mathrm{y}_{2}$, is

$$
\mathrm{y}_{2}=\mathrm{a}_{21} * \mathrm{x}_{1}+\mathrm{a}_{22} * \mathrm{x}_{2}+\ldots+\mathrm{a}_{2 \mathrm{p}} * \mathrm{x}_{\mathrm{p}}
$$

such that the variance of $y_{2}$ is maximized subject to the constraint that the squared weights of the second weight vector $\left(a_{21}, a_{22} \ldots a_{2 p}\right)$ is equal to one:

$$
\sum_{i=1}^{p} a_{2 i}^{2}=1
$$

Here, $\mathrm{y}_{2}$ is orthogonal to $\mathrm{y}_{1}$ so that

$$
\sum_{i=1}^{p} a_{1 i} a_{2 i}=0
$$

Hence, $\mathrm{y}_{2}$ has the second largest sum of squared correlation with the original variables. As successive components are extracted the variance of the principal components gets smaller. The first two principal components have the highest possible sum of squared correlation with the original variables. Therefore,

$$
\sum_{i=1}^{p} R^{2}{ }_{x i . y 1, y 2}
$$


is maximized by extracting the first two principal components. This is a measure of the explanatory power of the components. 3

\section{Component Scores}

The principal components are an exact mathematical transformation of the raw variables. If the objective is a simple summary of the information contained in the raw data, without recourse to any underlying factor analytical model, the use of component scores is desirable. It is possible to represent the components exactly from the combination of raw variables. The scores are obtained by combining the raw variables with weights that are proportional to their component loadings. Thus if $b_{i j}$ is the component loading of the $j$ th variable on the ith. component and $\lambda_{\mathrm{i}}$ is the associated eigenvalue then the component score is given by $b_{i j} / \lambda_{i}\left(=w_{i}\right)$.

The purpose of dividing by the eigenvalue is to assure that the resulting index has a variance equal to 1 . In our case the component scores have been used for determining the weight of each of the raw variables in constructing a composite Environment Degradation Index (EDI) for the ith country and, similarly, for other countries.

$$
E D I_{i}=\sum_{j=1}^{5} w_{j} \cdot x_{j i}
$$

$$
\text { where } \mathrm{j}=1,3,4 \text { and } 5 \text {. }
$$

\section{Component Extraction}

\footnotetext{
${ }^{23}$ See the Appendix for further treatment of the method of PCA.
} 
The first two components define the 'plane of closest fit'. The first three components define the three-dimensional hyperplane. As more and more components are extracted the measure of the explanatory power would increase. But this would lead to the number components approaching the number of variables. In which case, the entire variance is explained but the purpose of reducing the dimensionality is defeated. It is necessary to strike a balance between parsimony and explanatory power. To decide the optimal number of components to be extracted the literature has discussed three decision rules.

1. Kaiser's criterion, Kaiser (1960) ;

2. Jolliffe's criterion, Jolliffe (1972); and

3. Cattell's criterion, Cattell (1966).

The most frequently used decision rule is Kaiser's criterion. According to this rule, if a principal component of a correlation matrix has an eigenvalue whose value is less than one, it ought to be dropped because the variance of a single standardized variable is one. Hence, the component contains less information than the variable. By this count, however, small but significant components are ignored there may be a loss of information. Jolliffe feels that the Kaiser's rule discards too much information. On the basis of simulation studies he arrives at a cutoff point of $\lambda=0.7$. Cattell proposes the use of a Scree Plot ${ }^{4}$ for determining the number of components to be extracted. The plot charts eigenvalues against principal components as a visual aid for deciding. Principal components can be extracted up to the point of inflexion where the Scree Plot falls acutely and thereafter it flattens out. If $k$ is the discrete point on the $\mathrm{x}$-axis, at which this flattening takes place, then $k$ is the number of components to be retained.

\footnotetext{
${ }^{24}$ The scree plots are not included in this paper but are available on request from the corresponding author.
} 
If Kaiser's criterion were to be employed in our case then only the first two components would have to be extracted. Alternatively, two more components could have been considered for extraction, according to the Jolliffe's criterion. This would take the number of extracted components very close to the number of variables. However, the third component had a latent root that was very close to one $(0.89)$ and therefore, it could be included. Even according to (the Scree plot) Cattell's criterion three components were to be retained because after the third component the plot flattens out. The subsequent eigenvalues do not fall precipitately. Thus $k$ - the most appropriate number of principal components in our case is- three.

\section{Rotation of Principal Components}

An orthogonal rotation is a shift to a new set of co-ordinate axes in the sub-space spanned by the principal components. Sometimes the unrotated principal components cannot be readily interpreted. Therefore, often the components are rotated so as to achieve a more conceptually appealing or a simpler interpretation of the components. This may not alter the correlation matrix. The most popular orthogonal rotation procedure is Kaiser's Varimax rotation (Kaiser (1958)). The co-ordinate axes are rotated so as to maximize the Varimax criterion, which maximizes the sum of variances of the squared loadings within each column of the loading matrix. Both the unrotated and rotated solutions explain exactly the same amount of variation in the variables. The choice between them hinges upon the interpretative power of each solution. The original principal components maximize the sum of the squared loadings while the Varimax solution maximizes a 
variance-like function of the loading with the component. The component scores (both rotated and unrotated), with respect to the first component can be portrayed $\frac{25}{a s}$ :

\begin{tabular}{|l|l|l|}
\hline Variable Name & $\begin{array}{l}\text { Component Score } \\
\text { (unrotated) }\end{array}$ & $\begin{array}{l}\text { Component Score } \\
\text { (Rotated) }\end{array}$ \\
\hline PCFWW & 0.301 & -0.083 \\
\hline CENTFWW & 0.243 & -0.062 \\
\hline PAPCM & 0.299 & 0.520 \\
\hline PCCO2 & 0.383 & 0.390 \\
\hline CO2SH & 0.237 & 0.495 \\
\hline DEFOR & -0.270 & 0.102 \\
\hline
\end{tabular}

The problem with the unrotated component ${ }^{6}$ scores is that, except for DEFOR, the component scores are almost equal. Therefore, the resulting index would behave like an unweighted one. It defeats the purpose of undertaking this elaborate empirical procedure, that was meant to develop an exact, representative and composite.

\section{Construction of the Index.}

Once the number of retained principal components is determined and the rotated component scores are obtained, the choice lies between using the principal components as such or selecting certain sub-set of variables from amongst the larger set of variables. Jolliffe (1972) proposes selecting one variable to represent each of the retained principal components. The variable that has the highest loading on a component is chosen to represent that component, provided that it has not already been chosen. In such a case, the

\footnotetext{
${ }^{25}$ For the complete PCA results see Tables T1 to T7.
} 
variable with the next largest loading is chosen. The procedure starts with the largest principal component and proceeds to the smallest retained component. An alternative approach is to delete variables by using the discarded principal components. Starting with the smallest discarded component, the variable with the largest weight on that component is deleted. Then the variable with the largest loading on the second smallest component would be discarded. If the variable has previously been discarded, then the variable with the next highest loading would be discarded. This procedure continues up to the largest such discarded component.

We used the procedure of deleting variables, as outlined above, and were able to narrow down the number of variables from six to four. However, the principal components have themselves not been directly used. After having discarded two variables, viz., the second (CENTFWW) and the sixth (DEFOR)), the rest of already divided by the eigenvalue of the first component Hence, the Environmental Degradation index for the ith country is given as:

$$
E D I_{i}=\sum_{j=1}^{5} w_{j} \cdot x_{j i}
$$

where;

$\mathrm{w}_{\mathrm{j}}=\mathrm{j}$ th component score, $\mathrm{x}_{\mathrm{ji}}=$ value of the $\mathrm{j}$ th variable for the $\mathrm{i}$ th country; and $\mathrm{j}=1,3,4$ and 5 ; while Global environmental degradation is given by -

\footnotetext{
${ }^{26}$ Complete results from the unrotated component are not provided here to conserve space. These are,
} 
$G E D=\sum_{i=1}^{174} E D I_{i}$

This index is original, representative and complete.

One criterion that can be used for evaluating the efficiency of a sub-set of variables that represent a total set of variables is the total amount of variation that the sub-set explains. This can be obtained by adding the sum of the variances for the retained variables to the sum of the variation that they explain in each of the discarded variables. For determining this, the following formula due to Jolliffe (1986) was used:

Total variation $\operatorname{explanied}=n_{r}+\sum_{i \in d} R_{i, r}^{2}$

where the set $d$ consists of 1 and 6 .

Here, $n_{r}$ stands for the number of retained variables, $R_{i, r}^{2}$ stands for the squared multiple correlation of the ith discarded variable with the $r$ retained variables. This is obtained by regressing each of the two discarded variables on the four retained variables. The number of retained variables is added because each of the retained variables explains its own variation (variance $=1$ ). The measure $\mathrm{R}_{\mathrm{i}, \mathrm{r}}^{2}$ is added over the number of discarded variables because it represents the variation in the discarded variables explained by the retained variables.

\section{Identification of Outliers}

however, available from the corresponding author. 
Principal component analysis can be used for detecting outlying observations. The first two principal components account for a major portion of the total variation. These two components are orthogonal. If the two components are represented on the two axes, the respective observations would cluster along either axis. Outliers on the other hand, would deviate from the normal pattern of clustering. In this method the first principal component is measured along the $Y$ - axis and is plotted against the second component, measured on the $X$ - axis. Due to such a plotting, the outliers would stand out (Wilkinson (1986)). We employed a variation of this method to study outliers. The first two components were plotted simultaneously in the two-dimensional space with each of the points being labeled with the respective country names. Further, the entire set of data was partitioned into three human development classes - high, low and medium. Two advantages obtained. One, the clarity of each of the graphs improved vastly because of a relative dispersal of the data points of different countries. Two, the emerging pattern of clusters clearly establish the modal level of the data points in each of the development classes. The latter advantage, especially brings out the relative importance of the three development classes vis-à-vis environment degradation. As a part of this analysis, influential observations were sought to be identified. Here it is necessary to distinguish between outliers and influential observations. While an outlier might merely stand out in the given two-dimensional space, it may not necessarily structurally affect the component scores. That is to say that such cases would only represent a larger scale without radically affecting the component scores. On the other hand, there could be certain observations which are both outliers as well as influential observations. In such cases, the behavior of the particular country would significantly affect the overall component 
scores. There could be another type of country that is not an outlier but may be expected to have an influence. These patterns and influences have been studied and are reported in Figures 1 to 3.

\section{Figures 1 to 3 here.}

\section{Results}

The results of the foregoing empirical exercise can be analyzed under the following heads:

1. Composite Index and Development Classes.

2. Global Environmental Kuznets Curve.

3. Outliers and Influential Observations.

4. Distribution of Environment Degradation.

\section{Composite Index and Development Classes}

There were certain methodological considerations while employing principal component analysis for the purpose of developing a composite index. If the variances of different raw variables do not differ significantly and if the metric is the same for all variables, it is recommended that the covariance matrix should be employed rather than the correlation matrix. Our six variables (and the resultant data in the $174 \times 6$ data matrix) were not of the same metric (although there were not any major differences). Secondly, the initial descriptive statistics reveal that the variances are also quite different. The highest variance was that of CENTFWW $\left((325.2709)^{2}\right)$ and the lowest was that of DEFOR $\left((1.2596)^{2}\right)$. Thirdly, if the correlation between the variables were to be high it could be expected that a few principal components (two) could adequately represent all the variables. The highest correlation was between PCFWW and CENTFWW but only 0.377 
in absolute value. This is reflected in the initial eigenvalue of the first PC and the relatively low variation explained by it $\left(1.944\left(\lambda_{1}\right) / 6\right.$ (number of variables)), that is, 32.397 percent of the total variation in all the six variables. Hence, the correlation matrix was used and not the co-variance matrix.

In Tables 9 to 11, the details of the HDI Ranks, the EDI, the EDI rank and the difference between the two ranks are given. Even a cursory glance clearly brings out the patent inverse relationship between HDI Ranks and EDI ranks. For instance, in the case of USA the HDI Rank is 3, its EDI is 88.28 and its EDI Rank is 173 (out of 174)! The negative sign prefixing the 'diffrank' signify the slide down in the EDI rank in comparison to the HDI rank. The EDI of USA is two and a half times the average EDI prevalent in the High Human Development (HHD) country-class. It is about 11 times the average EDI of Medium Human Development (MHD) countries and 210 times that of Low Human Development countries. The average drop in rank in the HHD countries is 125 (ranks) (i.e., average HDI Rank - EDI rank). The inverse relationship is further reinforced by the negative correlation between HDI and EDI ranks (-0.727). The HDI in the MHD country class was only 3.01. And the gain in rank was 14.72 . The negative correlation is vindicated in this case too but the quantum is less $(-0.55)$. In the case of the Low Human Development (LHD) countries the correlation turns into a mild positive value. The EDI is extremely low and on an average the gain in rank is 121 .

\section{Tables 9 to 11 here.}

\section{Outliers and Influential Observations}

For determining whether a particular country is merely an outlier or it significantly affects the overall structure and magnitude of the component scores, the scores were re- 
estimated, by the set procedure outlined above, in each of the following cases, by eliminating, by turns, each of the individual countries listed below:

1. USA - outlier and large developed market economy.

2. Russia - vast country, an outlier and a non-market, declining economy.

3. China - outlier, vast, populous and non-market developing economy.

4. Finland - outlier (though) small and developed market economy.

5. Japan - small market economy, developed and populous and an outlier.

6. India - large, populous, mixed developing economy, not a significant outlier.

The inclusion of each of the above countries is justified on the grounds noted.

Each country is a deviant in some sense or the other. Table 12 contains results on seven sets of component scores - one overall and six individual sets. The brackets contain the percentage change in the component scores due to omission of the particular country. The sign of the component scores of all the variables were retained in the case of all countries, except the USA. In this case, all the signs became the opposite, except PCCO2, that remained positive. Secondly, in percentage terms the change induced in the component scores was recorded in each case. Here, again the influence that USA had on all the variables far outstripped that of all other countries put together. The total influence of USA terms was 2297 percent, while even netting for negative values. The average influence of USA over six variables was 382.83 percent per variable. Even the least influence, relating to PCCO2, was 70.5 percent. The effect of USA on CENTFWW was the maximum - over 982 percent. The next most influential country was China. The variable PCFWW was the most affected by China, whose effect was, nevertheless, was a mere 19.27 percent. The average influence of China, similarly, was only 3.5 percent. In 
all other case and for all other countries the average influence was less that unity. In the case of India, the average influence was just over 1 percent per variable. These results are collated in Table 12.

\section{Table 12 here.}

\section{A Global Environmental Kuznets Curve}

Now we revert back to the core questions? Does a GEKC exist? What is its shape? The study of correlation reveals that in the case of the HHD countries there is negative correlation between and HDI and EDI Ranks. Further, in the case of LHD countries the relationship becomes positive. In between the two ends the relationship is mildly negative. This is characteristic of a quadratic relationship. To further test the nature of the relationship a set of regressions were run. First, a linear function was fitted and it revealed a negative slope that was not highly significant. Subsequently we fitted a cubic

$$
E D I_{i}=\beta_{0}+\beta_{1} H D I_{i}+\beta_{2}(H D I)^{2}+\beta_{3}(H D I)^{3}+\varepsilon_{i}
$$

On plotting $\mathrm{EDI}_{\mathrm{i}}$ against $\mathrm{HDI}_{\mathrm{i}}$ ranks and by creating a predicted cubic trend line we obtain a classic cubic fit. Even though the quadratic had a high explanatory power (of around 0.66 ) it could not exceed the cubic fit. The adjusted $\mathrm{R}^{2}$ for the cubic case is 0.764. All the coefficients are highly significant. $\left(\beta_{1}=-2.1, \beta_{2}=0.0195 \& \beta_{3}=-5.7668 \mathrm{E}-\right.$ 05). The pattern clearly reveals a negatively sloped cubic GEKC with a characteristic inverted $N$. The first 50 countries account for almost the entire area under the cubic curve. Between the points 50 and 100 the cubic fit marginally dips into the negative 
quadrant. Thereafter, it rises a little above the $\mathrm{X}$-axis and plummets below the $\mathrm{X}$-axis again. This is portrayed in Table 13 and Figure 4.

\section{Table 13 and Figure 4 here.}

\section{Distribution of Environment Degradation}

On the basis of the constructed EDI, a histogram and an Ogive curve were mapped. The index was classified in class intervals of five units each, beginning from zero and going up to the highest class, that was beyond hundred. But it must be pointed out that the class up to zero was an open-ended class in the sense that it included negative value indices pertaining to a large number of low HDI rank countries. The lowest value was -1.99 . On the other hand, the highest class was also open-ended; being greater than hundred, but it consisted of only one high HDI rank country (Finland). For arriving at the area under the curve, normally, the mid-point of the class interval was taken and multiplied by the number of countries. The relative area in a given class interval was the ratio of the area in a particular class divided by the total area. However, since the lowest class extended below zero, for this class the mid-point was taken to be 0.5 . This way the total area (in relative terms) was greater than one, while the total (net) area adjusted for the lowest class (below zero) was equal to one.

Out of a total of 174 countries, 23 countries constituting over 13 percent had negative indices. In terms of the area under the Ogive curve, however, this class accounted for around -1 percent. The modal class (with EDI between 0-5) that had 92 countries, representing about 53 percent of the total number of countries, accounted for only 18.73 percent of the GED. The first three classes consisting of 131 countries, that is, 75.28 per cent of the countries, together accounted for 23.4 percent of the total 
degradation. The next three classes, having 20 countries (or 11.5 percent of the countries), were responsible for about 16 percent. An astounding 50 percent ratio of the degradation was accounted for by just 22 countries (that is 12.65 percent of the countries) in the higher bracket, save the highest. The highest class individually accounted for 10 percent. Two-thirds of the countries had indices that were either negative or did not exceed 5. The index of three-fourths of the countries did not exceed 10. Hence, only the top one-fourth was responsible for raising the index from 10 to over 100. In fact, it must be noted that the lowest class was responsible for pulling down global degradation in absolute terms. These results are reported in Table 14 and Figure 5.

\section{Table 14 and Figure 5 here.}

\section{Composite Index and Development Classes}

For constructing the composite index four of the six variables were retained. A measure of the explanatory power of the index is the percentage of the total variation explained. The multiple correlation coefficients pertaining to the discarded variable DEFOR obtained by regressing it on the retained variables is 0.32 . Similarly, in the case of CENTFWW the multiple correlation coefficient was 0.47 . Hence the total variation

explained was 4 (one each for each of the retained variables) and $(0.32)^{2}+(0.47)^{2}$ for the discarded variables. These add up to 4.326. Hence the explained variation is $(4.326 / 6) * 100$, i.e. $72.10 \%$. On the other hand if the three retained principal components were to be used the total variation explained would have been $68.75 \%$. The respective means of the first two components for the three country classes have been reported along with the standard error of the mean. This enables testing the statistical significance of the mean values with the help of t-tests. The reported $t$ statistics of the first component that 
denotes the maximum influence, clearly show that the high country class has a significant impact on the environment that enhances the degradation. The medium country class has a significant negative impact while the low country class has a highly significant negative impact. The second component denotes the minimum influence. In this case, the high and medium classes do not display significant mean values. Tables 15 to 17 summarize the results for the mean values of the principal components. The mean values of the first two principal components and tests for their significance are reported in Table 18.

\section{Tables 15 to 18 here.}

\section{Conclusions - EKC and Global Environmental Management}

This paper has articulated the necessity of emphasizing the absolute level of environmental degradation in different countries as a guide to understanding the links between such degradation and economic development. We argue the case for developing an aggregate index of such degradation and relating it to a better measure (in comparison to per capita income) of economic development, viz. the HDI. In this paper we have put forward a methodology that would achieve this end. This has necessitated the use of principal components analysis to extract information and for forming an index of global environmental degradation. This index has then been related to the HDI.

Some other conclusions of the paper are as follows. First, there seems to be an inverse link between HDI ranks and EDI ranks. Second, the US alone has a highly disproportionate (adverse) influence on all the six environmental degradation indicators. Therefore, in the design of a regulatory mechanism for global environmental management this factor would have to be recognized explicitly. 
In the extant literature there is no consensus about the empirical basis for the GED. All along the two contending views only try to verify whether or not an inverted $U$ shaped EKC exists. Even if we agree that an inverted $U$ shaped EKC does not exist it does not follow that an inverted $N$ shaped global EKC exists. Our study argues precisely that an inverted $N$ shaped global EKC does indeed exist and provides empirical support for this position. In the process we have found extreme inequalities in the contribution of low, medium and high country groups to GED with the low country group effectively ameliorating GED and the high group countries exacerbating it.

In a scenario where there is no consensus about even the state of global environmental degradation, there is an obvious need for an institution for the purposes of understanding and managing the global environment. A pertinent question to ask is: why the existing world bodies cannot handle this task? Steven (1993) points out that the WTO is not the appropriate body for dealing with environmental issues. Amongst other things it is felt that trade negotiations (of the WTO) are closed-door affairs and do not involve democratic institutions that are concerned with the environment in the process of negotiations (Shrybman (1990)). Secondly, the provisions of WTO imply a movement towards dismantling non-tariff barriers (NTBS), whereas environmental considerations may deem them necessary. For instance developing countries might want to protect their countries against trade in toxic waste. In this sense, as the deadline for dismantling controls is approaching fast, the need for an alternative world body that manages the global environment is also becoming increasingly imminent. While Esty (1994) and Runge (1994) provide an elementary basis for a prospective body like the World Environment Organization (WEO). Jha and Whalley (2000) and Whalley (1998) have 
done the pioneering work that provides the rationale, the institutional form and the possible manner of implementation of the WEO.

There are several important implications of the results of this paper and a few of these may be mentioned here. First, it provides a framework for assessing the current state of environmental degradation and its distribution world-wide. Thus, it would provide important inputs to a global agency like a WEO that would be interested in monitoring environmental degradation and its geographical distribution. Second, by relating such degradation to HDI, this relationship would highlight the contributions of countries with different levels of human development and assist in assigning liability. The contention that GED is essentially 'caused' by a certain type of development characteristic of high (income) development countries is borne out by the results of this study.

These conclusions clarify that the first and foremost concern of global environmental management, and the WEO, should be to reckon with the inequality in global environment degradation and its intrinsic relationship with inequalities across countries in levels of economic development. 


\section{References}

Antweiler,W.et.al.(1998) Is free trade good for the environment, Cambridge, Mass, NBER.

Arrow,K.et.al.(1995) "Economic Growth Carrying Capacity and the Environment" Science, vol. 286, pp.520-521.

Bartelle, B. (1994) "The High Cost of Turing Green" The Wall Street Journal, September 14.

Beckerman, W. (1992) "Economic Growth and the Environment: Whose Growth! Whose Environment?" World Development, vol. 20, pp. 481-496.

Beckerman, W. (1993) "The Environmental Limits to Growth: A Fresh Look" in H.

Giersch (ed.) Economic Progress and Environmental Concerns, Berlin, Springer.

Bergh,J. et.al.(1998) "Models of Individual Behaviour and the Implications for

Environmental Policy" World Congress of Environmental and Resource Economics, Venice, pp. 28.

Bernstam, M. (1991) The Wealth of Nations and the Environment, London, Institute for Economic Affairs

Birdsall, N. and D.Wheeler (1991) “Openness Reduces Industrial in Latin America: The Missing Pollution Haven Effect" Symposium of International Trade and the Environment, Washington, World Bank.

Broad,R.(1994) “The Poor and the Environment: Friends or Foe?” World Development, vol.22, pp. 811-822.

Cattell, R. et.al. (1966) "The Scree Test for the Number of Factors" Mutlivariate Behavioral Research, vol., pp. 245-276. 
Cole, M.(2000) Trade Liberalization Economic Growth and the Environment, Cheltenham, Edward Elgar.

Daly, H.E. (1977) Steady State Economics: The Economics of Bio-Physical Equilibrium and Moral Growth, San Francisco. W.H. Freeman.

Daly,H. and K.Townsend(1993) Valuing the Earth: Economics, Ecology and Ethics, Cambridge, Mass., MIT Press.

de Bruyn,S.(1997) Explaining the Environmental Kuznets Curve: Structural Change and International Agreements in Reducing Sulphur Emissions" Environment and Development Economics, vol. 2, pp. 485-504.

de Bruyn S. and J. Opshoor (1997) "Developments in the Throughput-Income Relationship: Theoretical and Empirical Observations”, Ecological Economics, vol. 20, pp. 255-268.

de Bruyn,S. et.al.(1998) "Economic Growth and Emissions: Reconsidering the Emipirical Basis of Environmental Kuznets Curve", Ecological Economics, vol.25, pp.161-175.

Ekins, P.(1993) "Limitis to Growth and Sustainable Development: Grappling with Ecological Realities", Ecological Economics, vol.8, pp. 269-288.

Ekins,P.(1997) "The Kuznets Curve for the Environment and Economic Growth:

Examining the Evidence", Environment and Planning, vol.29.

Esty,D.(1994) Greening the GATT: Trade Environment and the Future, Washington, Institute for International Economics.

GATT(1992) “Trade and Environment”, International Trade, Geneva, GATT.

Georgescu-Roegen, N.(1971) The Entropy Law and The Economic Process, Cambridge, Mass., Harvard University Press. 
Grossman, G. and A. Krueger (1995) "Economic Growth and the Environment" Quarterly Journal of Economics, vol. 110, pp. 353-377.

Hall, C. et.al.(1986) Energy and Resource Quality: The Ecology of the Economic Process, New York, John Wiley.

Harbaugh,W.et.al.(2000) Re-Examining the Empirical Evidence for an Environmental Kuznets Curve, Working Paper 7711, Cambridge, Mass, NBER.

Hettige,H. et.al.(1992) "The Toxic Intensity of Industrial Production: Global Patterns, Trends and Trade Policy", American Economic Review, vol. 82, pp.478-481.

Hettige,H.(1997) Industrial Pollution in Economic Development: Kuznets Revisited, Washington, World Bank.

Holtz-Eakin,D. and T.Selden(1992) Stoking the fires? CO2 Emissions and Economic Growth, Cambridge, Mass, NBER.

Hotelling, H. (1933) “Analysis of Complex Statistical Variables in Two Principal Components" Journal of Educational Psychology, vol.24, pp.417-441.

Janicke,M. et.al.(1989) "Economic Structure of Environmental Impacts: East-West Comparisons", The Environmentalist, vol. 19, pp.171-182.

Janson, C. (1969) “Some Problems of Ecological Factor Analysis" in Dogan, M. and S. Rokkan (ed.) Quantitative Ecological Analysis in Social Sciences, Cambridge, Mass., MIT Press..

Jha, R. and K. Bhanu Murthy (2000) "Sustainability: Property Rights, Behavior and Economic Growth", Papers and Proceedings of the World Congress on Measuring and Managing Sustainable Development, August. (Also Social Science Research Network Library). 
Jha, R. and J. Whalley (2000) “The Environmental Regime in Developing Countries"

in Carlo Carraro and Gilbert E. Metcalf, Editors Behavioral and Distributional Effects of

Environmental Policy, Chicago: University of Chicago for NBER

Jolliffe, I. (1972) “Discarding Variables in Principal Component Analysis" Applied

Statistics, vol.21, pp. 160-173.

Jolliffe, I. (1986) Principal Component Analysis, New York: Springer Verlag.

Kaiser, H. (1958) “The Varimax Criterion for Analytical Rotation in Factor Analysis"

Psychometrika, vol.23, pp.187-200.

Kaiser, H. (1960) “The Application of Electronic Computers to Factor Analysis”

Educational and Psychological Measurement, vol.20, pp. 141-151.

Kuznets, S.(1955) "Economic Growth and Income Inequality”, American Economic

Review, vol.49, pp.1-28.

Lewis-Beck, M. (1994) (ed.) Factor Analysis and Related Techniques, New Delhi: Sage Toppan.

Lopez, R. (1994) “The Environment as a Factor of Production: The Effect of Economic Growth and Trade Liberalization”, Journal of Environmental Economics and Management, vol.27, pp.163-184.

Moomaw,W. and G.Unruh(1997) “Are Environmental Kuznets Curves Misleading Us?

The Case of CO2 Emissions", Environment and Development Economics, vol.2, pp.451.

Panayotou,T.(1993) "Demystifying the Environmental Kuznets Curve: Turning a Black

Box into a Policy Tool”, Environment and Development Economics, vol.2, pp. 465-484.

Pearce,D.(1993) Blueprint Three: Measuring Sustainable Development, London,

Earthscan. 
Pezzy,J.(1989) Economic Analysis of Sustainable Growth and Sustainable Development, Washington, World Bank.

Rees,W.(1990) “The Ecology of Sustainable Development”, The Ecologist, vol.20, no. 1, pp.18-23.

Rothman,D.(1998) "Environmental Kuznets Curve: Real Progress or Passing the buck? A Case for Consumption Based Approaches”, Ecological Economics, vol.25, pp. 177-194. Runge,C.(1994) Freer Trade, Protected Environment: Balancing Trade Liberalization with Environmental Interests, New York, Council for Foreign Relations.

Ruttan,V.(1994) “Constraints on the Design of Sustainable Systems of Agriculture”, Ecological Economics, vol. 10, pp. 209-219.

Seldon, T. and D. Song (1994) "Environmental Quality and Development: Is there a Kuznets Curve for Air Pollution Emissions?" Journal of Environmental Economics and Management, vol.27, pp.147-162.

Shafik, N. and S. Bandopadhyay (1992) Economic Growth and Environmental Quality: Time Series and Cross-country Evidence" Background Paper for the World Development Report 1992, World Bank: Washington, D.C. Oxford University Press. Shyrbman,S.(1990) "International Trade and the Environment: An Environmental Assessment of GATT", The Ecologist, vol. 20, no.1.

Stagl,S.(1999) Delinking Economic Growth from Environmental Degradation: A Literature Survey on the Environmental Kuznets Curve Hypothesis, Vienna University Working Paper, Vienna. 
Stern, D.(1998) "Is There an Environmental Kuznets Curve for Sulfur? An Analysis of Bias in Environmental Kuznets Curve Estimation”, World Congress on Environmental Economics, Venezia, pp. 27.

Stern,D. et.al.(1996) "Economic Growth and Environmental Degradation: The Environmental Kuznets Curve and Sustainable Development”, World Development, vol. 24, pp. 1151-1160.

Steven,C.(1993) "Harmonization, Trade and the Environment", International Environmental Affairs, vol.5, no. 1, pp. 42-49.

Suri,V.and D.Chapman(1998) "Economic Growth Trade and Energy: Implications for The Environmental Kuznets Curve”, Ecological Economics, vol.25, pp.147-160.

UNDP (1999) Human Development Report, New York.

WCED(1987)(Brundtland Report) Our Common Future, Oxford, OUP.

Wilkinson, L. (1986) The System for Statistics, Evanston, Illinois:Systat.

World Bank(1992) World Development Report: Development and The Environment, Oxford, Oxford University Press. 


\section{Appendix}

\section{Basic Analytics of Principal Components}

This appendix discusses some basic issues in Principal Components Analysis. For a fuller treatment see Jolliffe (1986), Lewis-Beck (1994) and Hotelling (1933).

A principal component $\left(\mathrm{y}_{1}\right)$ is a linear composite of $p$ random variables $\left(\mathrm{x}_{\mathrm{i}}\right)$ :

$$
y_{1}=\sum_{i=1}^{p} a_{1 i} x_{i}
$$

Its variance is

$$
\sum_{i=1}^{p} \sum_{j=1}^{p} a_{i} a_{j} \sigma_{i j}
$$

with $\sigma_{\mathrm{ij}}$ being the covariance of $\mathrm{x}_{\mathrm{i}}$ and $\mathrm{x}_{\mathrm{j}}$.

If $a$ is a vector of variable weights and $C$ is the covariance matrix then PCA finds the weight vector such that it maximizes

$a^{\prime}$ Ca subject to

$$
\sum_{i=1}^{p} a_{i}^{2}=1
$$

If we have a set of $n$ observations on these $p$ variables then we can find the largest principal component of $R$ (the correlation matrix) after converting these random variables into standard normal variates as the weight vector $\left(\mathrm{a}_{11}, \mathrm{a}_{12}, \ldots, \mathrm{a}_{1 \mathrm{p}}\right)$ that maximizes the variance of

$\sum_{i=1}^{p} a_{1 i} x_{i}$ subject to $\sum_{i=1}^{p} a_{1 i}{ }^{2}=1$

Similarly for the second and third largest components such that

$\sum_{i=1}^{p} a_{1 i} a_{3 i}=\sum_{i=1}^{p} a_{2 i} a_{3 i}=0$ 
The sum of the variances of the principal components is equal to the sum of the variances of the original variables. Now, since there would be as many principal components as the number of variables we must have:

$$
\sum_{i=1}^{p} \lambda_{i}=\sum_{i=1}^{p} \sigma_{i}^{2}
$$

With standardized variables this would reduce to:

$\sum_{i=1}^{p} \lambda_{i}=p$

Hence the proportion of variance accounted for by $k$ principal components is

$\sum_{i=1}^{k} \lambda_{i} / p$

A generalization of the above would result in:

$\mathrm{Ra}=\lambda \mathrm{a}$, where $\lambda$ is the eigenvalue of the correlation matrix $R$ and $a$ is the eigenvector. 
Table T1

Descriptive Statistics

\begin{tabular}{|l|l|l|l|}
\hline & Mean & Std. Deviation & Analysis N \\
\hline Mean(PCFWW,2) & 6.0057 & 7.3429 & 174 \\
\hline Mean(CENTFWW,2) & 74.3076 & 325.2709 & 174 \\
\hline Mean(PAPCPM,2) & 17.7491 & 36.0513 & 174 \\
\hline Mean(PCCO2,20 & 4.6217 & 6.5794 & 174 \\
\hline Mean(CO2SHARE,2) & 0.5353 & 2.1212 & 174 \\
\hline Mean(DEFOR,2) & 0.5170 & 1.2596 & 174 \\
\hline
\end{tabular}

Table T2

Correlation Matrix

\begin{tabular}{|l|l|l|l|l|l|l|}
\hline & $\begin{array}{l}\text { Mean(PC } \\
\text { FWW,2) }\end{array}$ & $\begin{array}{l}\text { Mean(CE } \\
\text { NTFWW, } \\
2\end{array}$ & $\begin{array}{l}\text { Mean(PA } \\
\text { PCPM,2) }\end{array}$ & $\begin{array}{l}\text { Mean(PC } \\
\text { CO2,20 }\end{array}$ & $\begin{array}{l}\text { Mean(CO } \\
\text { 2SHARE, } \\
\text { 2) }\end{array}$ & $\begin{array}{l}\text { Mean(DE } \\
\text { FOR,2) }\end{array}$ \\
\hline $\begin{array}{l}\text { Mean(PC } \\
\text { FWW,2) }\end{array}$ & 1.000 & 0.377 & 0.059 & 0.21 & 0.124 & -0.241 \\
\hline $\begin{array}{l}\text { Mean(CE } \\
\text { NTFWW, } \\
\text { 2) }\end{array}$ & 0.377 & 1.000 & -0.042 & 0.294 & -0.018 & -0.073 \\
\hline $\begin{array}{l}\text { Mean(PA } \\
\text { PCPM,2) }\end{array}$ & 0.059 & -0.042 & 1.000 & 0.400 & 0.289 & -0.197 \\
\hline $\begin{array}{l}\text { Mean(PC } \\
\text { CO2,20 }\end{array}$ & 0.210 & 0.294 & 0.400 & 1.000 & 0.204 & -0.218 \\
\hline $\begin{array}{l}\text { Mean(CO } \\
\text { 2SHARE, } \\
\text { 2) }\end{array}$ & 0.124 & -0.018 & 0.289 & 0.204 & 1.000 & -0.95 \\
\hline $\begin{array}{l}\text { Mean(DE } \\
\text { FOR,2) }\end{array}$ & -0.241 & -0.073 & -0.197 & -0.218 & -0.95 & 1.000 \\
\hline
\end{tabular}




\section{Communalities}

Table T3

\begin{tabular}{|l|l|}
\hline & \multicolumn{1}{|c|}{ Initial } \\
\hline MEAN(PCFWW,2) & 1.000 \\
\hline MEAN(CENTFWW,2) & 1.000 \\
\hline MEAN(PAPCPM,2) & 1.000 \\
\hline MEAN(PCCO2,2) & 1.000 \\
\hline MEAN(CO2SHARE,2) & 1.000 \\
\hline MEAN(DEFOR,2) & 1.000 \\
\hline
\end{tabular}

Table T4

Total Variance Explained

\begin{tabular}{|l|l|l|l|l|l|l|}
\hline & \multicolumn{3}{|l|}{ Initial Eigenvalues } & \multicolumn{3}{l|}{ Rotation Sum of Squares Loadings } \\
\hline $\begin{array}{l}\text { Compone } \\
\text { nt }\end{array}$ & Total & $\begin{array}{l}\% \text { of } \\
\text { variance }\end{array}$ & $\begin{array}{l}\text { Cumulativ } \\
\text { e (\%) }\end{array}$ & Total & $\begin{array}{l}\% \text { of } \\
\text { Variance }\end{array}$ & $\begin{array}{l}\text { Cumulativ } \\
\text { e } \\
(\%)\end{array}$ \\
\hline 1 & 1.944 & 32.397 & 32.397 & 1.558 & 25.961 & 25.961 \\
\hline 2 & 1.290 & 21.501 & 53.898 & 1.495 & 24.925 & 50.886 \\
\hline 3 & 0.891 & 14.854 & 68.751 & 1.072 & 17.866 & 68.751 \\
\hline 4 & 0.826 & 13.760 & 82.511 & & & \\
\hline 5 & 0.582 & 9.692 & 92.203 & & & \\
\hline 6 & 0.468 & 7.797 & 100.00 & & & \\
\hline
\end{tabular}

Table T5

\section{Rotated Component Matrix ${ }^{27}$}

\begin{tabular}{|l|l|l|l|}
\hline & \multicolumn{3}{|c|}{ Component } \\
\hline & 1 & 2 & 3 \\
\hline MEAN(PCFWW,2) & 0.004318 & 0.708 & -0.324 \\
\hline MEAN(CENTFWW,2) & -0.001671 & 0.885 & 0.103 \\
\hline MEAN(PAPCPM,2) & 0.796 & -0.008274 & -0.197 \\
\hline MEAN(PCCO2,2) & 0.641 & 0.443 & -0.009529 \\
\hline MEAN(CO2SHARE,2) & 0.706 & -0.001308 & 0.004529 \\
\hline MEAN(DEFOR,2) & -0.108 & -0.00948 & 0.952 \\
\hline
\end{tabular}

Extraction Method: Principal Component Analysis

Rotation Method: Varimax with Kaiser Normalization

\footnotetext{
${ }^{27}$ Rotation converged in 5 iterations.
} 
Table T6

\section{Component Transformation Matrix}

\begin{tabular}{|l|l|l|l|}
\hline Component & 1 & 2 & 3 \\
\hline 1 & 0.689 & 0.595 & -0.414 \\
\hline 2 & -0.648 & 0.762 & 0.017 \\
\hline 3 & 0.326 & 0.256 & 0.910 \\
\hline
\end{tabular}

Table T7

\section{Component Score Coefficient Matrix}

\begin{tabular}{|l|l|l|l|}
\hline & \multicolumn{3}{|c|}{ Component } \\
\hline & 1 & 2 & 3 \\
\hline MEAN(PCFWW,2) & -0.083 & 0.449 & -0.219 \\
\hline MEAN(CENTFWW,2) & -0.062 & 0.641 & 0.231 \\
\hline MEAN(PAPCPM,2) & 0.520 & -0.149 & -0.071 \\
\hline MEAN(PCCO2,2) & 0.390 & 0.249 & 0.081 \\
\hline MEAN(CO2SHARE,2) & 0.495 & -0.058 & 0.169 \\
\hline MEAN(DEFOR,2) & 0.102 & 0.080 & 0.936 \\
\hline
\end{tabular}

Extraction Method: Principal Component Analysis

Rotation Method: Varimax with Kaiser Normalization 